\title{
The impact of apurinic-apyrimidinic endonuclease I on hepatocyte immuno-inflammatory factors and cell apoptosis
}

\author{
Tatsuo Sawakami ${ }^{1, \S}$, Zhipeng Sun ${ }^{2, \S, *}$, Yoshinori Inagaki ${ }^{1}$, Kiyoshi Hasegawa ${ }^{1}$, Wei Tang ${ }^{1,3}$, \\ Guangzhong $\mathrm{Xu}^{2}$, Nengwei Zhang ${ }^{2, *}$ \\ ${ }^{1}$ Division of Hepato-Biliary-Pancreatic Surgery, Department of Surgery, Graduate School of Medicine, The University of Tokyo, \\ Tokyo. Japan; \\ ${ }^{2}$ Oncology Surgery Department, Beijing Shijitan Hospital, Capital Medical University (Peking University Ninth School of \\ Clinical Medicine), Beijing. China; \\ ${ }^{3}$ National Center for Global Health and Medicine, Tokyo, Japan.
}

\begin{abstract}
Summary To explore the effect of apurinic-apyrimidinic endonuclease I (APE-1) on hepatocyte immune inflammatory factors and cell apoptosis. The gene expression profiles of peripheral blood of patients with or without immune tolerance after liver transplantation were obtained from the Gene Expression Omnibus (GEO). Differentially expressed genes were analyzed with a program in the $R$ language, and the APE-1 gene was identified as a gene related to immune tolerance of liver transplantation. Four APE-1 shRNA vectors were constructed in parallel and verified as correct using plasmid sequencing, real-time PCR, and Western blotting. An APE-1 overexpression vector was similarly constructed and verified as correct. The STRING website predicted the protein-protein interaction network of APE-1. ELISA was used to detect the effects of APE-1 silencing and overexpression on inflammatory cytokines IL-1 $\beta$, IL-10, TNF $\alpha$, and INF- $\gamma$ in the control group, APE-1-silenced group, and APE-1 overexpression group. Flow cytometry was used to detect apoptosis in each group. Forty differentially expressed genes related to immune tolerance after liver transplantation were screened, and the highly expressed gene APE-1 was selected. The best APE-1 shRNA_1 vector and APE-1 overexpression vector were obtained. APE-1 is predicted to interact with ANP32A, FEN1, HMGB2, LIG1, MUTYH, NTHL1, OGG1, PCNA, POLB, SET, and other proteins. APE-1 silencing resulted in a significant increase in the expression of the inflammatory factors IL-1 $\beta$, IL-10, TNF $\alpha$, and INF- $\gamma$ in L-02 cells. In contrast, the expression of APE-1 led to a significant decrease in the expression of inflammatory factors. APE-1 silencing significantly increased the rate of apoptosis of L-02 cells, and APE-1 overexpression resulted in a significant decrease in the rate of apoptosis of L-02 cells. In conclusion APE-1 affects the expression of inflammatory factors and apoptosis in L-02 cells, so it may be a key gene in immune tolerance of liver transplantation.
\end{abstract}

Keywords: Liver transplantation; immune tolerance; apurinic-apyrimidinic endonuclease 1 (APE-1); small hairpin RNA (shRNA); cell apoptosis

\section{Introduction}

Liver transplantation has become an effective method for the treatment of end-stage liver disease. Patients

\footnotetext{
${ }^{\S}$ These authors contributed equally to this work.

*Address correspondence to:

Drs. Zhipeng Sun \& Nengwei Zhang, Oncology Surgery Department, Beijing Shijitan Hospital, Capital Medical University (Peking University Ninth School of Clinical Medicine), Beijing 100038, China.

E-mail: sunzhipeng56084@163.com, zhangnw1@sohu.com
}

have a 1 -year and 5-year survival rate of $85 \%$ and $70 \%$, respectively. However, the incidence of primary graft non-function is still as high as $15 \%$. Liver transplantation faces the challenge of immune rejection (1). Over the past few years, an increasing number of studies have shown that peripheral blood microarray detection can provide biomarkers of transcriptions that help to identify immune tolerance after liver transplantation. The current study was based on transcriptome data from patients with immune tolerance after liver transplantation. Once apurinic-apyrimidinic endonuclease 1 (apurinic- 
apyrimidinic endonuclease I, or APE-1) was identified as a gene related to immune tolerance after liver transplantation, APE-1 shRNA and an overexpression vector were constructed to further explore its impact on the expression of immunoinflammatory factors and apoptosis in the L-02 human normal liver cell line.

\section{Materials and Methods}

\subsection{Data acquisition and main reagents}

\subsubsection{Data acquisition and cell sources}

GSE11881 raw data were downloaded from the Gene Expression Omnibus (GEO) (https://www.ncbi.nlm. nih.gov/geo/query/acc.cgi? acc $=$ GSE11881). The L-02 human normal liver cell line was purchased from the Peking Union Basic Research Institute.

\subsubsection{Main reagents}

The total RNA extraction reagent TRIzol, the SYBR ${ }^{\circledR}$ PrimeScript ${ }^{\mathrm{TM}}$ RT-PCR Kit, and mRNA SYBR Green fluorescent PCR reagents were purchased from Takara, Japan. A RIPA lysate for protein extraction, a BCA protein concentration test kit, and SDS-PAGE gel reagents were purchased from Jiangsu Beyotime. IL$1 \beta$, IL-10, TNF $\alpha$, and INF- $\gamma$ ELISA kits were purchased from Beijing Berui Technology. The cell transfection reagent Lipo 2000 was purchased from Invitrogen, USA. APE1 antibody and GAPDH antibody were purchased from Abcam, UK. An Annexin V-FITC/PI double-stain apoptosis detection kit was purchased from Jiangsu Keygen Biotech. The pLK0.1-puro vector was purchased from Sigma Aldrich China.

\subsection{Procedure}

\subsubsection{Screening for differentially expressed genes} related to immune tolerance after liver transplantation

After gene expression values for each sample were homogenized using a program in the R language, the statistical significance of the differences was set at $<0.05$, and patients with or without immune tolerance after liver transplantation were screened for differentially expressed genes.

\subsubsection{Construction of an APE-1 shRNA vector}

Four pairs of APE-1 shRNA sequences were designed and synthesized, as shown in Table 1. Annealing of shRNA primers. The primers were diluted with ultrapure water to $10 \mu \mathrm{M}$. After the upstream primer was mixed with the downstream primer at a ratio of 1:1, the mixture was denatured at $100^{\circ} \mathrm{C}$ for $10 \mathrm{~min}$ in a water bath and then cooled down naturally to room temperature. Selection of the pLKO.1 plasmid vector (https://www. sigmaaldrich.com/content/dam/sigma-aldrich/docs/ Sigma/Bulletin/shc001bul.pdf) and linearized pLKO.1 plasmid vector: $30 \mu \mathrm{l}$ and $150 \mathrm{ng} / \mu \mathrm{L}$ of $\mathrm{pLKO} .1$ were prepared for digestion. A pLKO.1 vector doubledigestion system was constructed. After digestion at $37^{\circ} \mathrm{C}$ for $3 \mathrm{~h}$, the plasmid was recovered, purified, and dissolved in $30 \mu \mathrm{L}$ of TE buffer. The pLKO.1 plasmid was ligated into the vector and verified with sequencing.

\subsubsection{Cell transfection / Seeding cells}

L-02 cells were seeded onto 6 -well plates (1x106 cells/ well) 1 day prior to transfection. Transfection started when cell confluence reached $90 \%$, and the media were changed $2 \mathrm{~h}$ before transfection. Seven-pointfive $\mu \mathrm{g}$ of plasmid DNA was added to $250 \mu \mathrm{L}$ of OPTIMEM media and mixed thoroughly. Then, $10 \mu \mathrm{l}$ of Lipo 2000 was added to another $250 \mu \mathrm{L}$ of OPTI-MEM and mixed gently. The mixture was allowed to stand at room temperature for $5 \mathrm{~min}$. The DNA suspension was mixed with the Lipo 2000 suspension, reaching a total volume of $500 \mu \mathrm{L}$. After gentle mixing, the mixture was allowed to stand at room temperature for $20 \mathrm{~min}$. After thorough mixing by shaking, the DNA and Lipo 2000 mixture was added to each well of the 6-well plates. The plates were placed in an incubator. After transfection for $6 \mathrm{~h}$, the media were changed ( $2 \mathrm{~mL}$ in each well). After transfection for $48 \mathrm{~h}$, the cells were harvested for detection of expression.

\subsubsection{Real-time PCR detection of APE-1 mRNA expression in L-02 cells}

Total cellular RNA was extracted using the Trizol

Table 1. Four pairs of APE-1 shRNA sequences in parallel

\begin{tabular}{ll}
\hline shRNA name & \multicolumn{1}{c}{ Sequence information } \\
\hline shRNA_1-F & CCGGCAGAGAAATCTGCATTCTATTCTCGAGAATAGAATGCAGATTTCTCTGTTTTT \\
shRNA_1-R & AATTCAAAAACAGAGAAATCTGCATTCTATTCTCGAGAATAGAATGCAGATTTCTCTG \\
shRNA_2-F & CCGGGCCTGGACTCTCTCATCAATACTCGAGTATTGATGAGAGAGTCCAGGCTTTTT \\
shRNA_2-R & AATTCAAAAAGCCTGGACTCTCTCATCAATACTCGAGTATTGATGAGAGAGTCCAGGC \\
shRNA_3-F & CCGGCCACTCTCTGTTACCTGCATTCTCGAGAATGCAGGTAACAGAGAGTGGTTTTT \\
shRNA_3-R & AATTCAAAAACCACTCTCTGTTACCTGCATTCTCGAGAATGCAGGTAACAGAGAGTGG \\
shRNA_4-F & CCGGCCTGGATTAAGAAGAAAGGATCTCGAGATCCTTTCTTCTTAATCCAGGTTTTT \\
shRNA_4-R & AATTCAAAAACCTGGATTAAGAAGAAAGGATCTCGAGATCCTTTCTTCTTAATCCAGG \\
\hline
\end{tabular}


technique. Ten $\mu \mathrm{L}$ of RNA was used for reverse transcription. GAPDH was used as internal reference for relative quantitative analysis. The quantitative reaction system included $12.5 \mu \mathrm{L}$ of SYBR Premix Ex Taq, $1 \mu \mathrm{L}$ of PCR Forward Primer, $1 \mu \mathrm{L}$ of PCR Reverse Primer, 2 $\mu \mathrm{L}$ of cDNA template and $8.5 \mu \mathrm{L}$ of ddH2O, with a total volume of $25 \mu \mathrm{L}$. Three parallel wells were prepared for each sample. The amplification procedures were as follows: pre-denaturation at $95^{\circ} \mathrm{C}$ for $30 \mathrm{~s} ; 95^{\circ} \mathrm{C}$ for $5 \mathrm{~s}$ and $60^{\circ} \mathrm{C}$ for $20 \mathrm{~s}$, for a total of 40 cycles. The relative expression of APE-1 mRNA was calculated using the $2^{-\Delta \Delta \mathrm{Ct}}$ method.

\subsubsection{Determination of APE-1 protein expression in L-02} cells using Western blotting

Cells were collected to extract total proteins for quantification using the BCA method. SDS-PAGE gel electrophoresis: After the transfer, the membrane was placed in a blocking solution containing 5\% skim milk powder-TBST for blocking at room temperature for $1 \mathrm{~h}$. Diluted APE-1 and GADPH primary antibodies (1:800 dilution) were added and gently shaken at $4{ }^{\circ} \mathrm{C}$ overnight; then, the corresponding secondary antibody $(1: 5,000$ dilution) was added, and the mixture was incubated at $37^{\circ} \mathrm{C}$ for $1 \mathrm{~h}$ on a constant-temperature shaker. Color development for calculation of the relative expression of protein: The software ImageJ was used to calculate the gray value and APE1/GAPDH of APE1 expression.

\subsubsection{Detection of inflammatory factor expression using ELISA}

Concentrations of IL-1 $\beta$, IL-6, IL-10, TNF $\alpha$, INF- $\gamma$, and other inflammatory factors in the cell culture supernatant were determined in accordance with the ELISA kit instructions. The standard curves were plotted according to concentrations and OD values of the standards. Then, the concentration in a sample was calculated on the standard curve according to the OD values. The result was the relative level of expression.

\subsubsection{Detection of apoptosis using Annexin V-FITC/PI double labeling technique}

Cells in each group were collected, placed on a 100-mesh copper screen, and rubbed gently. After rinsing with normal saline, the samples were centrifuged at 2,500 r/ $\mathrm{min}$. The supernatant and cell debris were discarded, and then the cell suspension was collected. Flow cytometry was used for analysis based on the method described in instructions in the Annexin V-FITC/PI double-staining apoptosis detection kit.

\subsection{Statistical processing}

SPSS 17.0 was used for statistical analysis. Measurement data were expressed as mean $\pm \mathrm{SD}$, and inter-group differences were compared using one-way analysis of variance. If the inter-group difference was statistically significant, a $t$-test was used to further compare the difference between 2 groups. When $P<0.05$, the difference was statistically significant.

\section{Results}

\subsection{Screening for genes related to immune tolerance after liver transplantation}

Gene expression profiles of peripheral blood from 9 patients with immune tolerance after liver transplantation (GSM124753, GSM124834, GSM124836, GSM124845, GSM124851, GSM124853, GSM124646, GSM124850, GSM124648) and 8 patients without immune tolerance (GSM124863, GSM124833, GSM124575, GSM124748, GSM124838, GSM124840, GSM124842, GSM124675) in the GEO database were selected. As shown in Figure 1, screening yielded 40 significantly differentially expressed genes $(P<0.05)$. Previous studies by the current authors indicated that APE-1 protein can reduce immunogenicity in addition to the dual functions of redox and DNA repair. Immune tolerance of the donor liver resulted (8). The results of this microarray screening suggested that the APE-1 gene is still highly expressed in patients with immune tolerance after liver transplantation, which is consistent with results of previous studies. Therefore, the APE-1 gene was selected for subsequent study.

\subsection{Construction of an APE-1 ShRNA vector and evaluation of silencing efficiency}

Plasmid sequencing verified that the 4 APE-1 shRNAs

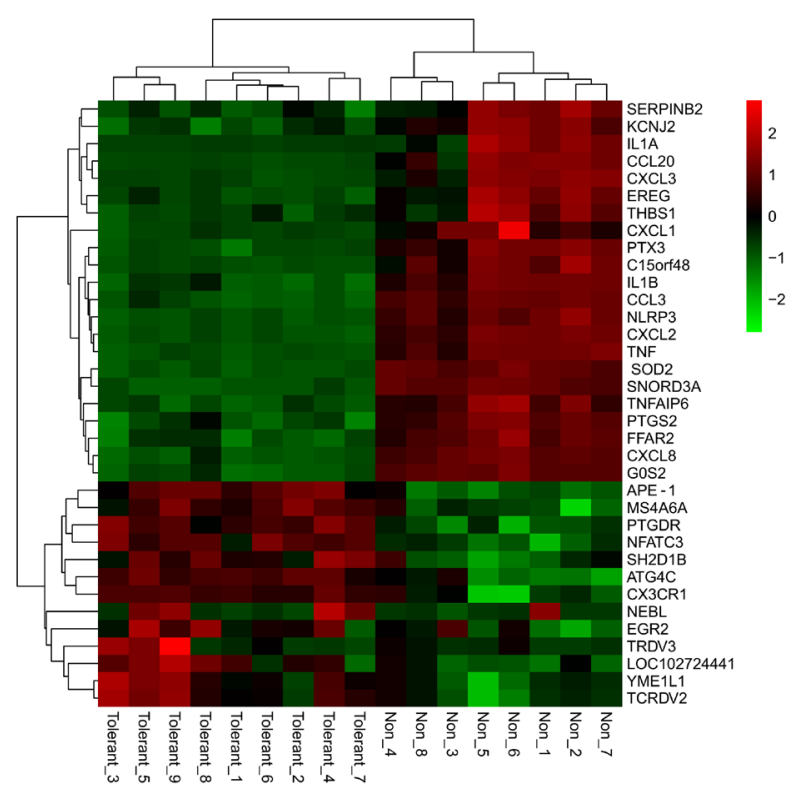

Figure 1. Screening for differentially expressed genes related to immune tolerance after liver transplantation. 


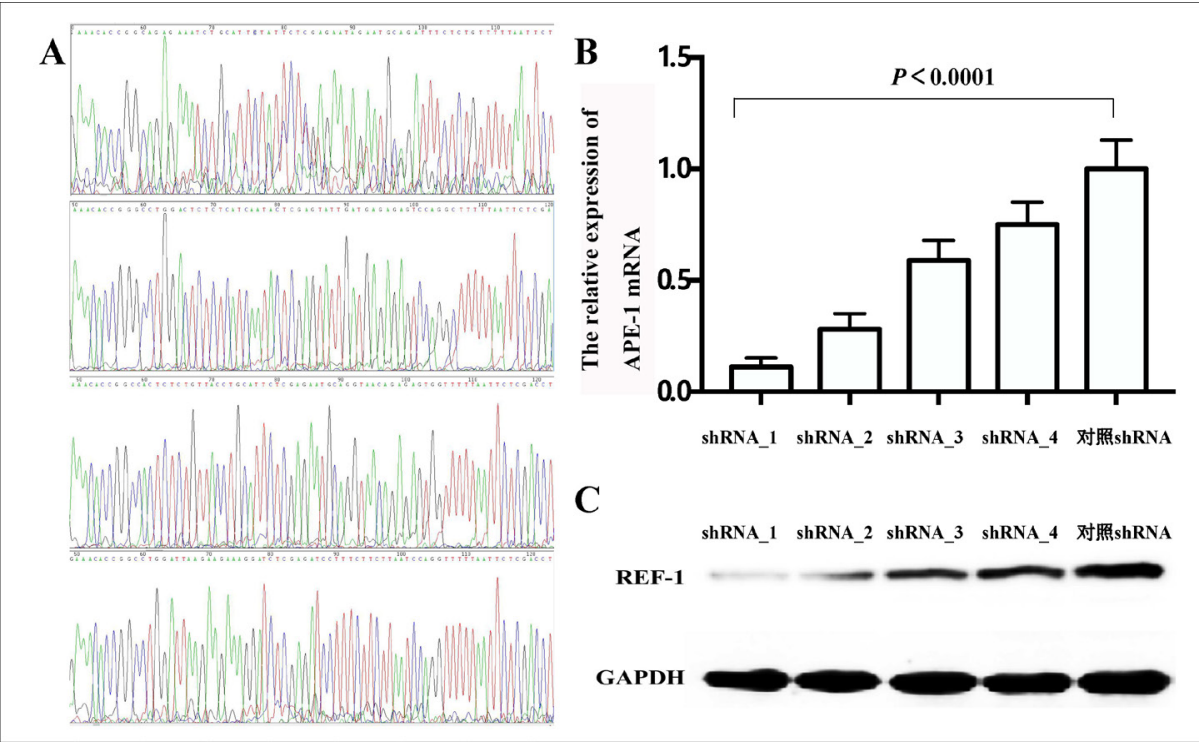

Figure 2. Construction of an APE-1 shRNA vector and evaluation of silencing efficiency.

A

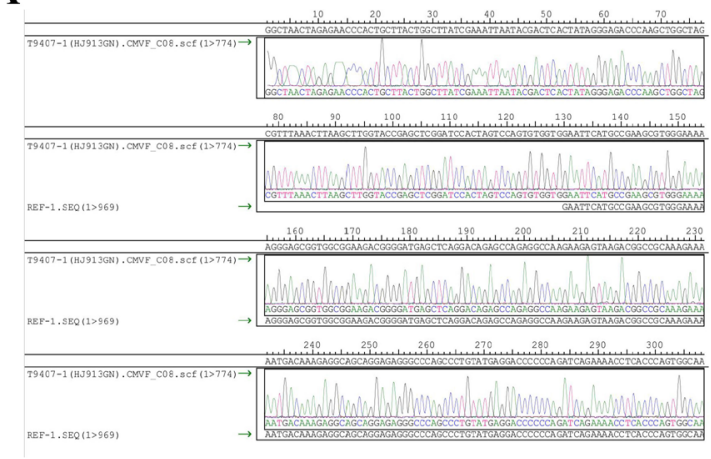

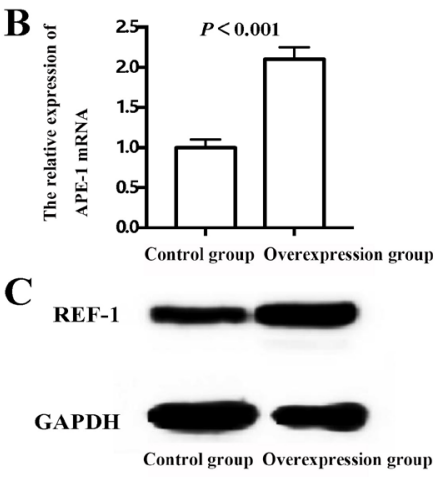

Figure 3. Construction of an APE-1 overexpression vector and evaluation of overexpression efficiency.

were successfully constructed in parallel, as shown in Figure 2A. After stable transfection of L-02 cells, realtime PCR was used to detect the expression of APE1 mRNA in hepatocytes. Results indicated that APE1 mRNA expression was significantly lower in cells transfected with the 4 APE- 1 shRNAs than in the control shRNA transfection group $(P<0.05)$, as shown in Figure $2 \mathrm{~B}$. When protein expression in each group was detected using Western blotting, the results were consistent with those of mRNA detection. APE-1 protein expression was significantly lower in each APE-1 shRNA group than in the control shRNA group (Figure 2C), demonstrating the effectiveness of the designed shRNA interference sequence. Silencing was most apparent with APE-1 shRNA_1, so it was used in subsequent experiments.

\subsection{Construction of an APE-1 overexpression vector and evaluation of overexpression efficiency}

Plasmid sequencing verified that the APE-1 overexpression vector was successfully constructed
(Figure 3A). After stable transfection of L-02 cells, real-time PCR was used to detect the expression of APE1 mRNA in hepatocytes. results indicated that mRNA expression was significantly higher in the APE1 overexpression group than in the control group ( $P$ $<0.05$, Figure 3B). The results of Western blotting were consistent with the level of mRNA detected. Protein expression was significantly higher in the APE1 overexpression group than in the control group (Figure $3 \mathrm{C}$ ), demonstrating that the APE-1 overexpression vector was successfully constructed.

\subsection{Prediction of the protein-protein interaction network of APE-1}

The protein-protein interaction network of APE-1 was predicted using the STRING website (Version: 10.5). APE-1 was found to potentially interact with proteins such as ANP32A, FEN1, HMGB2, LIG1, MUTYH, NTHL1, OGG1, PCNA, POLB, and SET (Figure 4A). Correlation coefficients are shown in Figure 4B. 
A

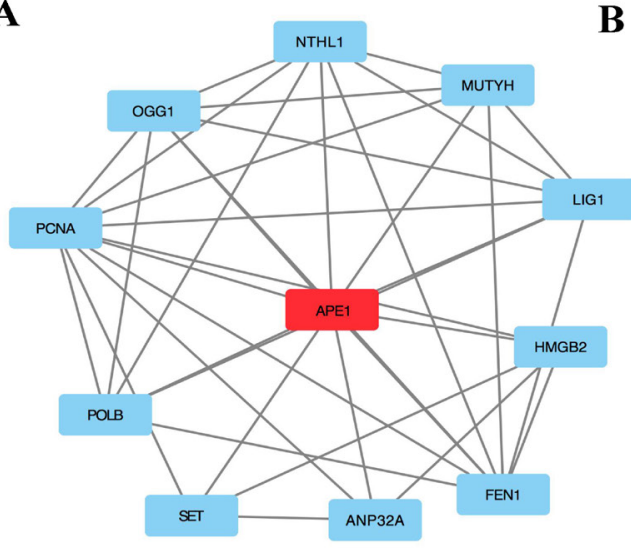

B

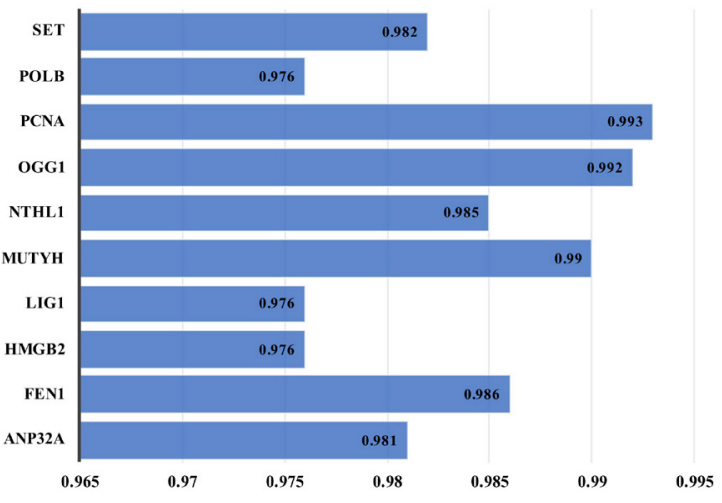

Figure 4. Prediction of the protein-protein interaction network of APE-1.

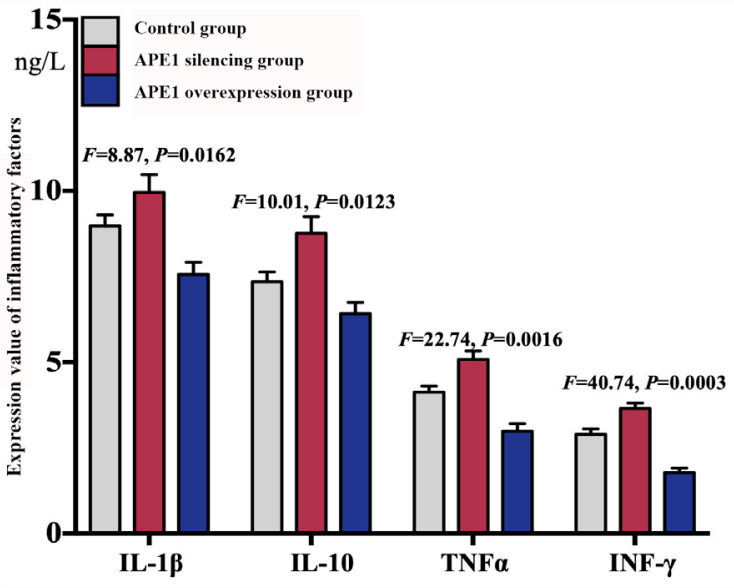

Figure 5. Impact of APE-1 silencing and overexpression on immune factors in L-02 cells.

\subsection{Impact of APE-1 silencing and overexpression on the expression of immune factors in hepatocytes}

ELISA was used to determine changes in expression of the inflammatory factors IL- $1 \beta$, IL-10, TNF $\alpha$, and INF- $\gamma$ in L-02 cells in the control group, the APE-1-silenced group, and the APE-1 overexpression group. Results indicated that the expression of inflammatory factors increased significantly in the APE-1-silenced group and decreased significantly in the APE-1 overexpression group (Figure 5).

\subsection{Impact of APE-1 silencing and overexpression on hepatocyte apoptosis}

The Annexin V-FITC/PI double-labeling technique was used to detect the apoptosis of L-02 cells in the control group, the APE-1-silenced group, and the APE1 overexpression group. The total rate of apoptosis was $13.24 \pm 0.234 \%, 19.75 \pm 0.398 \%$, and $10.61 \pm 0.366 \%$, respectively. One-way analysis of variance revealed significant differences among the groups $(F=192.6, P<$ 0.01 ). APE-1 silencing significantly increased the rate of apoptosis of L-02 cells while overexpression of APE-1 significantly decreased the rate of apoptosis of L-02 cells $(P<0.01)$ (Figure 6).

\section{Discussion}

Liver transplantation is the most effective means for the treatment of end-stage liver disease. Patients have a 1 -year and 5 -year postoperative survival rate of $85 \%$ and $70 \%$, respectively. However, liver transplant recipients still have a lower long-term life expectancy than the normal population. Immune tolerance is a topic of interest in the field of organ transplantation. Due to low selectivity, the currently available immunosuppressants inevitably cause extensive suppression of the recipient's immune system, which can induce infections and tumors while reducing the acceptance of the organ transplant. The adverse effects of immunosuppressants, including renal impairment, diabetes, cardiovascular diseases, and metabolic syndrome, are also important causes of a diminished quality of life and even death of recipients (2-3). The use of genetic technologies to induce immune tolerance is an important part of the current research in the field of organ transplantation, where the search for effective target genes is crucial.

Various types of new detection technologies emerging over the past few years have made clinical high-throughput detection possible, greatly facilitating advances in the study of potential target genes involved in immune tolerance. Using an oligonucleotide microarray to examine PBMC specimens from patients with immune tolerance and patients lacking immune tolerance, studies have found that some genes are overexpressed in peripheral $\gamma \delta \mathrm{T}$ cells and NK cells. Further studies have identified 3 gene combinations from 13 genes, resulting in an amazing accuracy in distinguishing between patients with immune tolerance and patients lacking immune tolerance with an error rate 


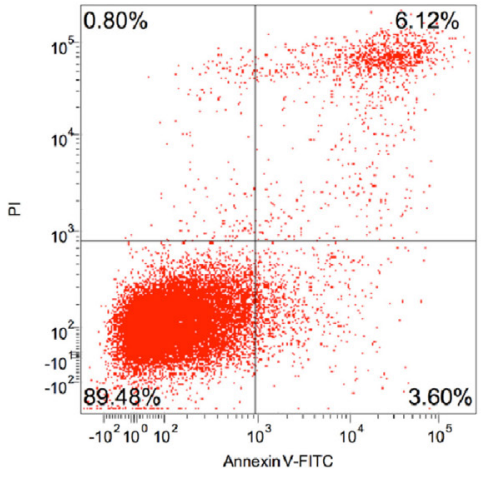

APE1 overexpression group

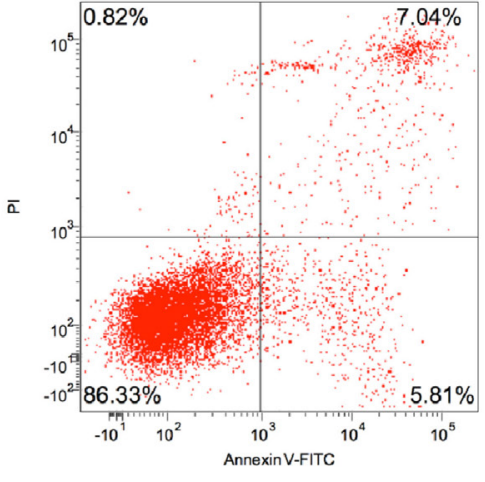

Control group

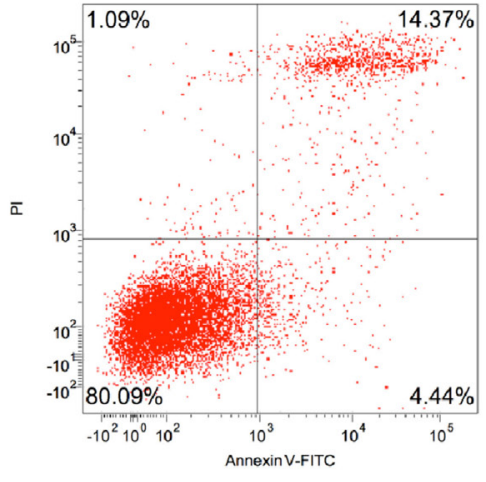

APE1 silencing group

Figure 6. Impact of APE-1 silencing and overexpression on the apoptosis of L-02 cells.

of merely $13-17 \%$. Pathological data from peripheral blood and liver transplants have been analyzed, revealing significant differences in gene transcription products between patients with immune tolerance and patients lacking immune tolerance (4). Some studies have found that the rate of immune tolerance was significantly higher in children than in adults, but the mechanisms differed in those sets of patients. In order to identify the target genes for immune tolerance in child and adult recipients of liver transplants, transcriptional profiles were obtained from 300 child and adult liver transplant recipients and normal tissues and blood samples. Results indicated that all 13 genes overexpressed in peripheral blood of patients with immune tolerance came from NK cells. The ERBB2, FEM1C, and SENP6 genes had a sensitivity of 1.00 , a specificity of 0.83 , and an area under the curve of $0.988(5)$.

In the current study, gene expression profiles of peripheral blood of patients with or without immune tolerance after liver transplantation were downloaded from the GEO. A program in the R language was used to analyze differentially expressed genes, and the APE-1 gene was identified as a gene related to immune tolerance after liver transplantation. An APE-1shRNA_1 silencing vector and an APE-1 overexpression vector were successfully constructed, revealing that APE-1 silencing resulted in significant up-regulation of the expression of the inflammatory factors IL-1 $\beta$, IL-10, TNF $\alpha$, and INF- $\gamma$ in L-02 cells. In contrast, overexpression of APE-1 resulted in significant down-regulation of inflammatory factor expression. Silencing of APE-1 expression significantly increased the apoptosis of L-02 cells while APE-1 overexpression resulted in significantly decreased apoptosis of L-02 cells, suggesting that changes in APE1 immunoreactivity may be related to apoptosis. APE-1 is able to excise and repair bases. Diminished ability to repair damaged DNA due to reduced APE-1 may be an important cause of increased apoptosis.

In addition, the current authors' team previously explored the phenomenon whereby overexpression of APE-1 protein enhanced immune escape of the graft.
Creation of an orthotopic liver transplantation model in rats indicated that hepatocyte apoptosis gradually declined and liver function gradually improved with potentiated APE-1 gene expression and accumulation of intracellular APE-1 protein (6-7). Multiple organ failure after liver transplantation was treated as an adverse reaction to immunosuppressants. The current authors' team found that multiple organ failure after liver transplantation in the established rat model mainly occurred in the liver and kidneys. Liver transplantation causes little damage to the lungs and intestines. APE1 protein plays a role in repairing renal injury after liver transplantation. When APE-1 protein expression is upregulated, renal function improved according to indices such as CREA and BUN, and the apoptosis of renal cells gradually decreased (8-9). Those changes in apoptosis were consistent with the results of the current study.

Predictions indicated that APE-1 potentially interacted with ANP32A, FEN1, HMGB2, LIG1, MUTYH, NTHL1, OGG1, PCNA, POLB, SET, and other proteins. These functional proteins are all involved in the development and progression of liver cancer and immune tolerance (10-11). PCNA, for example, is related to histological differentiation, tumor size, and prognosis for patients with hepatocellular carcinoma, and it is also involved in immune signaling pathways in liver cancer cells. ANP32A participates in the development and progression of liver cancer and is closely related to tumor prognosis. FEN1 is overexpressed in hepatocellular carcinoma tissues, and FEN1 overexpression is indicative of low tumor differentiation, ready metastasis, and a poor prognosis.

In summary, APE-1 affects the expression of inflammatory factors in L-02 cells and apoptosis; it may be the key gene for immune tolerance after liver transplantation.

\section{Acknowledgements}

This study was supported by the Beijing Municipal Education Commission general scientific research 
project (no. KM202010025008), Opening Foundation of Beijing Key Lab of Therapeutic Cancer Vaccines (no. 2019-JS04), the Foundation of Beijing Shijitan Hospital, Capital Medical University (no. 2019-C10), and grantsin-aid from the Ministry of Education, Science, Sports, and Culture of Japan.

\section{References}

1. Chen GH, Jiang N. Research hotspots and considerations of immune tolerance in liver transplantation. Organ Transplantation, 2017; 8:1-4.

2. Geng L, Huang JJ, Lin BY, Chen TC, Shen T, Zheng SS. Characteristics of recipients with complete immunosuppressant withdrawal after adult liver transplantation. Hepatobiliary Pancreat Dis Int. 2017; 16:437-439.

3. Hussaini T, Turgeon RD, Partovi N, Erb SR, Scudamore $\mathrm{CH}$, Yoshida EM. Immunosuppression practices in liver transplantation: A survey of North American centers. Exp Clin Transplant.2018; 16:550-553.

4. Bohne F, Martínez-Llordella M, Lozano JJ, et al. Intragraft expression of genes involved in iron homeostasis predicts the development of operational tolerance in human liver transplantation. J Clin Invest. 2012; 122:368382.

5. Li L, Wozniak LJ, Rodder S, Heish S, Talisetti A, Wang Q, Esquivel C, Cox K, Chen R, McDiarmid SV, Sarwal MM. A common peripheral blood gene set for diagnosis of operational tolerance in pediatric and adult liver transplantation. Am J Transplant. 2012; 12:1218-1228.

6. Zhang P, Du X, Sun ZP, Xu L. Expression of redox factor-1 in early injury period after liver transplantation in rat model. Cell Mol Immunol. 2009; 6:309-313.

7. Guo L, Haga S, Enosawa S, Naruse K, Harihara Y, Sugawara, Y. Improved hepatic regeneration with reduced injury by redox factor-1 in a rat small-sized liver transplant model. American journal of transplantation. 2004; 4:879-887.

8. Sun ZP, Zhu YB, Am BH, Gong K, Zhang NW. Redox factor-1 may mediate the repair of multiple organ injuries after liver transplantation. Chin Med J. 2013; 126:25042509.

9. Sun ZP, Am BH, Zhu B, Gong K, Zhang NW. Antiinducible costimulator immunotherapy inhibits accelerated and chronic cardiac allograft rejection in rats. Journal of Clinical Rehabilitative Tissue Engineering Research. 2010; 14:8347-8351. (in Chinese)

10. Yan W, Chen X. Research progress on the relationship between ANP32A and tumor. Acta Medicinae Sinica, 2017; 30:141-144

11. Yin R, Wu WX, Wang HH, Chen Y. Effects of FEN1 overexpression on the biological behaviors of hepatocellular carcinoma cells and the prognosis of patients. Cancer Research on Prevention and Treatment, 2016; 43:848-853.

(Received November 26, 2019; Revised December 14, 2019; Accepted December 16, 2019) 